2D dosimetry in a proton beam with a scintillating GEM detector

This article has been downloaded from IOPscience. Please scroll down to see the full text article.

2009 Phys. Med. Biol. 543755

(http://iopscience.iop.org/0031-9155/54/12/010)

View the table of contents for this issue, or go to the journal homepage for more

Download details:

IP Address: 131.180.130.109

The article was downloaded on 08/08/2011 at 10:11

Please note that terms and conditions apply. 


\title{
2D dosimetry in a proton beam with a scintillating GEM detector
}

\author{
E Seravalli ${ }^{1,3}$, M R de Boer ${ }^{1}$, F Geurink ${ }^{1}$, J Huizenga $^{1}$, R Kreuger $^{1}$, \\ J M Schippers ${ }^{2}$ and C W E van Eijk ${ }^{1}$ \\ ${ }^{1}$ Delft University of Technology, Faculty of Applied Sciences, Delft, The Netherlands \\ 2 Paul Scherrer Institute, Villigen, Switzerland \\ E-mail: e.seravalli@tudelft.nl
}

Received 19 December 2008, in final form 5 April 2009

Published 28 May 2009

Online at stacks.iop.org/PMB/54/3755

\begin{abstract}
A two-dimensional position-sensitive dosimetry system based on a scintillating gas detector is being developed for pre-treatment verification of dose distributions in particle therapy. The dosimetry system consists of a chamber filled with an $\mathrm{Ar} / \mathrm{CF}_{4}$ scintillating gas mixture, inside which two gas electron multiplier (GEM) structures are mounted (Seravalli et al 2008b Med. Phys. Biol. 53 4651-65). Photons emitted by the excited $\mathrm{Ar} / \mathrm{CF}_{4}$ gas molecules during the gas multiplication in the GEM holes are detected by a mirrorlens-CCD camera system. The intensity distribution of the measured light spot is proportional to the $2 \mathrm{D}$ dose distribution. In this work, we report on the characterization of the scintillating GEM detector in terms of those properties that are of particular importance in relative dose measurements, e.g. response reproducibility, dose dependence, dose rate dependence, spatial and time response, field size dependence, response uniformity. The experiments were performed in a $150 \mathrm{MeV}$ proton beam. We found that the detector response is very stable for measurements performed in succession $(\sigma=0.6 \%)$ and its response reproducibility over 2 days is about $5 \%$. The detector response was found to be linear with the dose in the range 0.05-19 Gy. No dose rate effects were observed between 1 and $16 \mathrm{~Gy} \mathrm{~min}^{-1}$ at the shallow depth of a water phantom and 2 and $38 \mathrm{~Gy} \mathrm{~min}^{-1}$ at the Bragg peak depth. No field size effects were observed in the range $120-3850 \mathrm{~mm}^{2}$. A signal rise and fall time of $2 \mu$ s was recorded and a spatial response of $\leqslant 1 \mathrm{~mm}$ was measured.
\end{abstract}

(Some figures in this article are in colour only in the electronic version) 3 Address for correspondence: Department of Radiation, Radio nuclides \& Reactors, Division RD\&M, Delft
University of Technology, Mekelweg 15, 2629 JB Delft, The Netherlands. 


\section{Introduction}

Particle therapy represents, today, the most promising radiotherapy technique for external tumor treatments. Charged particle beams show an increasing energy deposition with penetration distance leading to a maximum (the Bragg peak) near the end of the particle range. Behind this maximum, the energy deposition drops very fast within a few $\mathrm{mm}$. Consequently, the dose delivered by a charged particle beam is well localized in depth with a small lateral spread allowing a precise scanning of the tumor volume.

Conformal patient treatment plans are characterized by strong dose gradients at the treatment volume contour. Therefore, relative particle-therapy dosimetry requires systems that are characterized by high spatial resolution $(\leqslant 1 \mathrm{~mm}$ ) for (in-phantom) 2D and 3D dose distribution measurements. These systems should have a linear response over a large dynamic range and be able to deal with high intensity beams $\left(\sim 10^{9}\right.$ particles $\left.\left(\mathrm{cm}^{2} \mathrm{~s}\right)^{-1}\right)$.

The response of state-of-the-art 2D dosimeters (e.g. radiographic films (Butson et al 2003, Spielberger et al 2001, 2003) and scintillating screens (Boon et al 1998b, Safai et al 2004)) depends on the particle energy. A correction for this energy dependence cannot be applied when multiple proton energies are contributing to the local dose.

A gas-filled detector is expected to have a smaller energy dependence and a faster response (Seravalli 2008a) than radiographic films and scintillating screens. We are developing a position-sensitive scintillating gas detector based on GEMs (Sauli 1997) for relative 2D dose measurements in charged particle beams. A GEM is a copper clad thin kapton foil with a regular pattern of sub-millimeter holes. The light emitted by the electron-excited scintillating gas mixture molecules during the gas multiplication process in the GEM holes is detected by means of a mirror-lens-CCD camera system. The measured 2D light intensity distribution is proportional to the $2 \mathrm{D}$ distribution of the energy deposited in the sensitive volume by the beam. For measurement of 3D dose distribution, the scintillating GEM detector is mounted at the beam exit side of a water-bellows phantom, whose thickness can be varied in steps.

We have recently demonstrated that in an alpha particle beam (Seravalli et al 2007) and clinical carbon ion beam (Seravalli et al 2008b), the scintillating GEM detector response underestimation of the Bragg peak dose is much smaller than the one presented by state-ofthe-art 2D dosimeters with a millimeter resolution (e.g. Boon 1998a, Spielberger et al 2003). It has been shown (Fetal et al 2003, Timmer et al 2002) that a similar detector to the one described here has only a small signal underestimation in a proton Bragg peak. Therefore, the scintillating GEM detector is a promising device for 2D and 3D dose measurements, especially in the case of high LET beams for verifying treatment plans composed of several beam energies.

In this work, we report on the characterization of the scintillating GEM detector in terms of those properties that are of particular importance in relative dose measurements in (scanning) proton beams, e.g. response reproducibility and uniformity; dose, dose rate and field size dependence; spatial and time response.

In a $150 \mathrm{MeV}$ proton beam, the output of the scintillating GEM detector has been compared with a parallel plate air-filled ionization chamber response (our reference) and with the scintillating Lanex screen signal.

\section{Methods and materials}

\subsection{The scintillating GEM detector}

The detector consists of a $350 \times 350 \times 50 \mathrm{~mm}^{3}$ aluminum chamber continuously flushed $\left(91 \mathrm{~h}^{-1}\right)$ with an $\mathrm{Ar} / \mathrm{CF}_{4}$ gas mixture at $1 \mathrm{~atm}$. Inside the chamber, two cascaded 
$100 \times 100 \mathrm{~mm}^{2}$ GEMs, produced at CERN (Sauli 1997) and named GEM 1 and GEM 2 , have been mounted. The GEMs used in this work have double conical holes and are glued onto $1 \mathrm{~mm}$ thick $\mathrm{Al}$ frames. For a detailed description of the detector, we refer to (Seravalli et al 2008b).

A voltage of $370 \mathrm{~V}$ was used over each GEM. The electric field in the drift and transfer gap (Seravalli et al 2008b) was set respectively to $1 \mathrm{kV} \mathrm{cm}^{-1}$ and $1.5 \mathrm{kV} \mathrm{cm}^{-1}$. These settings guaranteed stable detector operation for the applied beam rates.

The data reported here were collected in two different measurement campaigns. In the first one, GEMs with big holes ( $80 \mu \mathrm{m}$ diameter, $140 \mu \mathrm{m}$ pitch) and $\mathrm{Ar}+6 \% \mathrm{CF}_{4}$ (percentage volume) were used to study the reproducibility, dose linearity, dose rate dependence and time response. During the second measurement campaign, GEMs with small holes $(60 \mu \mathrm{m}$ diameter, $90 \mu \mathrm{m}$ pitch) and $\mathrm{Ar}+8 \% \mathrm{CF}_{4}$ were used because we had learnt that with this configuration a brighter light signal, a higher electric signal and a better signal-to-noise ratio are obtained than that with $80 \mu \mathrm{m}$ diameter holes for identical measurement conditions (Seravalli et al $(2008 \mathrm{c})$ ). With the second detector configuration, we studied not only the spatial resolution but also the dose linearity again, to check if the improved signal-to-noise ratio would allow us to measure smaller doses with the same accuracy. Since in Seravalli et al (2008c), it is shown that both the GEM hole diameter and the percentage of $\mathrm{CF}_{4}$ influence the detector output, one cannot draw conclusions from the observed differences in the light intensity measured in this work. However, for the properties studied in this work, the relative light yield of these two detector configurations is not relevant.

The photons produced in the electron avalanches are detected by means of a low darkcurrent CCD camera coupled with a zoom lens (Seravalli et al 2008b and 2008c). The CCD signal per pixel is expressed in analog-to-digital units (ADU), 1 ADU being equivalent to 8.4 electrons' collected charge.

The focusing of the CCD camera on $\mathrm{GEM}_{2}$ is done by means of a $100 \mathrm{~mm}$ diameter transparent foil with a $10 \mathrm{~mm}$ pitch grid, temporarily mounted at the $\mathrm{GEM}_{2}$ location. The optical magnification factor of the whole setup is 0.041 so that 1 pixel $(9 \mu \mathrm{m} \times 9 \mu \mathrm{m})$ on the $\mathrm{CCD}$ is equivalent to $218 \mu \mathrm{m} \times 218 \mu \mathrm{m}$ at the position of $\mathrm{GEM}_{2}$.

Simultaneously to the light signal, the cathode and GEMs currents are measured for a better understanding of the detector operation. The currents are measured by means of nanoamperemeters connected in series with the high voltage line which supplies each GEM surface (Seravalli et al 2008b).

The temperature and pressure inside the detector were monitored by means of a sensor mounted on the detector chamber.

After 3600 Gy of proton irradiation, we have measured a $3 \%$ decrease in transmission of the glass exit window in the range $400-600 \mathrm{~nm}$. This is a known phenomenon in the literature (Holmes-Siedle and Adams 1993). The transmission decrease as a function of time might affect the light signal reproducibility. In the next detector prototype, the glass window will be replaced by a quartz window, the latter being more radiation resistant. See for example, Vukolov and Levin (2003).

\subsection{Experiment setup}

The detector has been irradiated in a steady $150 \mathrm{MeV}$ proton beam of the AGOR cyclotron at Kernfysisch Versneller Instituut, Groningen, the Netherlands (KVI 2008). A schematic of the beam line and the experimental setup is given in figure 1 . 


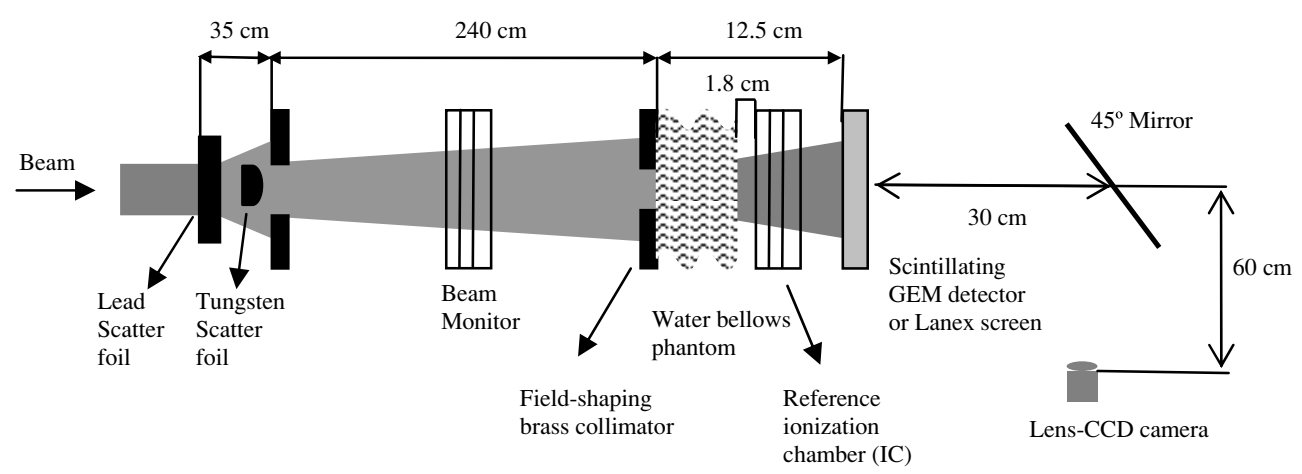

Figure 1. Schematic of the proton beam line and the experimental setup seen from above.

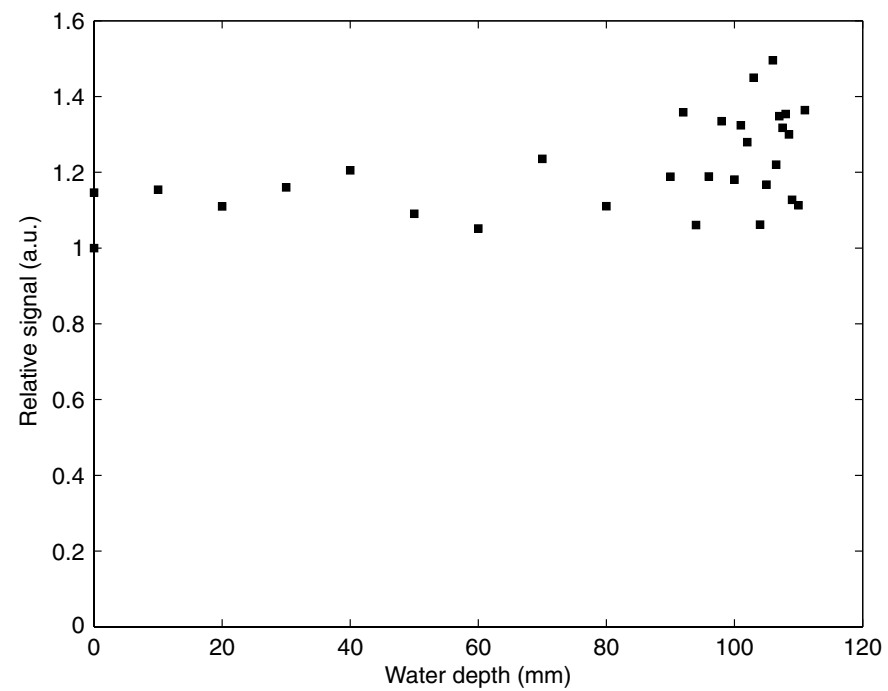

Figure 2. (bk3 - bk2) as a function of the water depth. The data are normalized to the (bk3 - bk2) value measured at 0 -wd (23 ADU).

An air-filled parallel plate ionization chamber was used as a beam monitor to register the beam intensity in MU s$~^{-1}$. The MU (monitor unit) has been calibrated in dose to water at the scintillating GEM detector location by means of a PTW23343 Markus chamber.

The dose rate was varied by changing the proton current in the cyclotron source. Experiments were performed at 0 -wd (see below for definition) in the dose rate range $1-16 \mathrm{~Gy} \mathrm{~min}{ }^{-1}\left(1 \mathrm{~Gy} \mathrm{~min}^{-1}=2 \times 10^{7}\right.$ particles $\left.\left(\mathrm{cm}^{2} \mathrm{~s}\right)^{-1}\right)$.

The field-shaping brass collimator determines the beam spot size at the entrance of a water-filled bellows phantom (figure 2). The field size was varied by appropriate field-shaping collimator shapes. Field sizes ranged from $1 \times 10 \mathrm{~mm}^{2}$ to $20 \times 20 \mathrm{~mm}^{2}$, and radii from $2.5 \mathrm{~mm}$ up to $40 \mathrm{~mm}$ were used for circular collimators.

The beam energy was degraded by means of a water-filled bellows phantom, whose thickness can be varied in steps of $\approx 0.05 \mathrm{~mm}$ from zero up to beyond the proton range. The minimum bellows phantom water depth $(0-w d)$ leaves $3 \mathrm{~cm}$ of plastic of the phantom in the 
beam. Bp-wd indicates the water depth at which the Bragg peak is measured by the detector. At $\mathrm{Bp}$-wd, the dose rate measured by means of an ionization chamber is about 3.5 times higher than that measured at $0-w d$.

Due to energy losses in the beam line components, air and the water phantom frame, the effective proton energy at 0 -wd was $121 \mathrm{MeV}$ if both scatter foils were inserted in the beam line, and $127 \mathrm{MeV}$ if only the first one was present.

The charge $q_{\text {IC }}$ of a second air-filled parallel plate ionization chamber (IC) has been used as a reference. As can be seen in figure 2, the scintillating GEM detector was placed behind the IC with respect to the beam direction.

For a single measurement, the beam is turned on at a particular beam intensity for a certain period of time. In that period, an amount of particles, corresponding to a predetermined amount of dose in water, is delivered to the scintillating GEM detector. The camera shutter is open and the emitted light is integrated on the CCD for the exposure time set. At the same time, the GEM detector electric signals and the beam monitor output, $I_{b}$, are sampled at $1 \mathrm{kHz}$.

For comparison, measurements with a Lanex scintillating screen were performed as well. The screen was fixed to a holder and the holder mounted on the plastic tube (black tube in figure 2), supporting the mirror and the CCD camera, instead of the scintillating GEM detector. The holder was fixed in such a way that the Lanex screen was at the same distance from the CCD camera as $\mathrm{GEM}_{2}$.

\subsection{Data analysis and background}

The obtained pictures are processed offline using Matlab routines to correct for backgrounds. The classification of background components that can be found in a picture is discussed in section 2.3.1.

2.3.1. Background components. In a picture $p$ taken for a certain exposure time $\Delta t_{\mathrm{e}}$ and for a certain dose $D$, the following background sources can be identified.

- The bias level or offset (bk0).

- It is a fixed contribution present in every picture, independent of the exposure time. It can be calculated from a picture, bk0, measured for the minimum CCD camera exposure time $(0.01 \mathrm{~s})$.

- The dark current (bk1 - bk0).

- It depends on the CCD camera temperature and on the exposure time. It can be quantified taking a picture, bk1, for the same exposure time $\Delta t_{e}$, and the same cooling temperature of $p$ but with the CCD camera shutter closed.

- Direct interaction of scattered beam radiation with the CCD camera chip that results in large signals in isolated pixels (spikes) (bk2 - bk1).

- This background can be reduced by properly shielding the CCD camera, e.g. by means of lead bricks. It can be measured by taking a picture, bk2, with radiation beam on and CCD camera shutter closed for $\Delta t_{\mathrm{e}}$ seconds and $D \mathrm{~Gy}$.

- Light emitted in the detector when the GEMs are off while $E_{\mathrm{d}}, E_{\mathrm{t}}$ and the light gap (the gap between $\mathrm{GEM}_{2}$ and the exit window) electric field is on (bk3 - bk2).

- This background component is due to the scintillation of the gas mixture in the light gap and/or scintillation in the glass exit window. It can be evaluated taking a picture, bk3, with radiation beam on, CCD camera shutter open for $\Delta t_{\mathrm{e}}$ seconds and $D$, with GEMs off and $E_{\mathrm{d}}, E_{\mathrm{t}}$, light gap electric field on. 
Table 1. Typical light intensity values of a picture $p$ measured at 0 -wd for $6 \mathrm{~Gy}, 30$ s exposure time and of the background sources.

\begin{tabular}{lc}
\hline & Light intensity (ADU/pixel) \\
\hline$p$ & 4806 \\
bk0 (bias level) & 1401 \\
bk1 - bk0 (dark current) & 32 \\
bk2 - bk1 (spikes) & 4 \\
bk3 - bk2 (gas/glass scintillation) & 23 \\
$p-$ bk1 & 3373 \\
$p-$ bk2 & 3369 \\
$p-$ bk3 & 3346 \\
\hline
\end{tabular}

The bias level and the dark current are radiation beam independent background sources, while the remaining background components are radiation beam related.

In table 1, typical light intensity values (in the beam center) of a picture $p$ (measured at 0 -wd, 6 Gy, 30 s exposure time) and of the background sources are depicted. The dark current (bk1 - bk0), the spikes (bk2 - bk1) and the gas/glass scintillation (bk3 - bk2) components are small $(<1 \%)$ compared to the light signal $(p-b k 3)$.

In principle, for a fixed exposure time $\Delta t_{e}$ and dose $D$ Gy bk $3>\mathrm{bk} 2>\mathrm{bk} 1>\mathrm{bk} 0$. It should be noted that when bk 3 is subtracted from $p$ all the other background components are compensated at once except for the spikes that can be filtered out subsequently with a median filter.

2.3.2. The background due to the light emitted when GEMs are off $(b k 3-b k 2)$. We have observed that (bk3 - bk2) does not scale with the dose along a depth-dose curve. In figure 2, (bk3 - bk2) is shown as a function of the water depth ${ }^{4}$. This kind of background is almost constant till about $90 \mathrm{~mm}$ and then increases and its value fluctuates around a higher level. The (bk3 - bk2) increase at the Bragg peak depth, $\sim 108 \mathrm{~mm}$, is not compatible with the expected peak to plateau ratio of 3.5 in this proton beam. This can be attributed to the fact that the scintillation of the exit window glass is quenched at the Bragg peak, a phenomenon that occurs in solid-state materials due to the increased ionization density of the proton tracks (Murray and Meyer 1961).

When the main signal is relatively small, which is the case for this background signal, the random spikes, which are not fully removed by a median filter, may contribute to the rather large fluctuations in figure 2. However, the importance of these fluctuations should not be overestimated, since for the typical pictures the 4 ADU spike contribution (bk2 - bk1) is small compared to the signal of $3300 \mathrm{ADU}(p-\mathrm{bk} 3)$.

We have found that for a fixed delivered dose and a fixed water depth, (bk3 - bk2) is independent of the electric field in the light gap within the experimental errors. Light gap gas scintillation would increase with a higher voltage. Together with what was observed in figure 2, this indicates that (bk3 - bk2) is mainly due to the exit window glass scintillation.

2.3.3. Analysis. First, bk3 is subtracted from the pictures because it does not scale with the dose along a depth-dose curve. Then, a 3-by-3 median filter (MathWorks 2007) is applied to the background-subtracted pictures in order to remove large signals on isolated pixels (spikes)

4 In order to measure bk3 as a function of the water depth, a picture was taken at 0-wd with GEMs off, drift and transfer gap electric field on and beam on. Then, the thickness of the water bellows phantom was increased in steps up to a value larger than the proton range, and for each step a picture was taken for the same conditions. 
created by the direct interaction of scattered radiation in the CCD and not compensated by subtraction of bk3.

The integrated light yield, $L$, has been calculated by integrating the background-corrected picture pixel values in ADU, after filtering, over a circular region of interest. The region of interest is chosen bigger than the beam spot recorded in a picture taken at the Bragg peak depth and it is kept constant for all the pictures.

We define as output current, $I_{\text {out }}$, the current flowing to the surface of $\mathrm{GEM}_{2}$ facing the exit window.

The $I_{\text {out }}$ offset, $I_{\text {offset }}$, is calculated taking the mean value over $N$ samples of $I_{\text {out }}\left(t_{i}\right)$ recorded before the beam starts $\left(t_{\mathrm{s}}\right)$. The output charge $q_{\text {out }}$ is evaluated summing the offset corrected $I_{\text {out }}$ values between $t_{\mathrm{s}}$ (beam starts) and $t_{\mathrm{f}}$ (beam stops) instants (Seravalli et al 2008b).

$L$ and $q_{\text {out }}$ are normalized to the integrated signal of $I_{\mathrm{b}}$, between $t_{\mathrm{s}}$ and $t_{\mathrm{f}}$, to correct for variations in the dose delivered.

The detector outputs are corrected for ambient pressure variations according to the procedure described in Seravalli et al (2008c).

\subsection{Uncertainties}

2.4.1. $q_{\text {out }}$ uncertainty. The experimental error of $q_{\text {out }}$ is quantified considering a set of measurements performed ten times in succession for the same measurement conditions yielding the standard deviation $(\sigma)$ around the mean value. For typical conditions $(6 \mathrm{~Gy}, 20 \mathrm{~s}$ beam on time, $11 \times 12 \mathrm{~mm}^{2}$ collimator, 0 -wd), we found a $q_{\text {out }}$ standard deviation of $0.5 \%$.

Beam fluctuations can be excluded as an error source because the data have been normalized to the beam monitor signal $I_{b}$. The $q_{\text {out }}$ uncertainty calculated by means of the error propagation formula for the samples of a single measurement is comparable to the experimentally found $\sigma$. Therefore, this error is related to the precision of the readout system used to record $I_{\text {out }}$.

2.4.2. L uncertainty. To quantify the $L$ experimental error, likewise the $q_{\text {out }}$ uncertainty, we determined the standard deviation $(\sigma)$ around the mean of a set of $L$ values, measured ten times in succession for the same conditions. For typical conditions ( $6 \mathrm{~Gy}, 30 \mathrm{~s}$ exposure time, 31397 pixels, 0 -wd), we found a standard deviation of $0.6 \%$.

It should be noted that $L$ is obtained integrating the pixel values over a region of interest involving 31397 pixels. The uncertainty in $L$ is thus much smaller than the variation of the signal of the individual pixels.

Beam fluctuations can be excluded as an error source because the data have been normalized to the integrated beam monitor signal $I_{\mathrm{b}}$.

An error $(\sigma)$ of the same order of magnitude was observed for ten Lanex measurements performed in succession. Therefore, the above mentioned $0.6 \%$ uncertainty is mainly due to the optical system used to readout the light photons and not to the scintillating GEM detector.

In the graphs shown in the following figures, the value of $3 \sigma$ is used as an error bar for $q_{\text {out }}$ and $L$ values. Error bars are only shown when graph markers are smaller than the experimental uncertainties.

\section{Results and discussion}

\subsection{Short-term response reproducibility}

The detector response short-term reproducibility (several days) was evaluated considering measurements performed at 0 -wd for the same conditions (GEMs with big holes, $\mathrm{Ar}+6 \%$ 

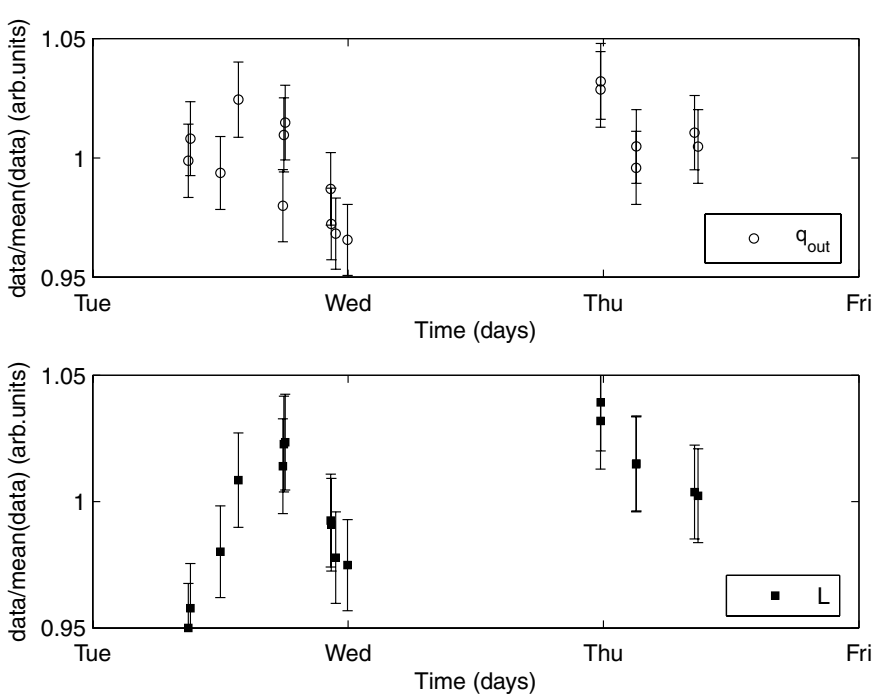

Figure 3. $q_{\text {out }}$ (top graph) and $L$ (bottom graph) as a function of the measurement time, in days. The data were measured for big hole GEMs in $\mathrm{Ar}+6 \% \mathrm{CF}_{4}$ under identical measurement conditions. On the $y$-axis, the ratios of the single data values and the average of all the data values are reported. Error bars represent $3 \sigma$.

$\mathrm{CF}_{4}, 11 \times 12 \mathrm{~mm}^{2}$ field size, $6 \mathrm{~Gy}$ ) at different moments during the beam time period. As can be seen in figure 3 , the $q_{\text {out }}$ and $L$ reproducibility over 2 days is about $5 \%$.

The causes of these detector response variations with time are not yet fully understood. Ambient pressure changes (Seravalli et al 2008c) and beam fluctuations are excluded because the data were compensated for them. Moreover, there is no systematic decrease in $L$ values due to a loss in transmission of the glass exit window (section 2.1).

The detector response variations can be related to the signal formation process because both detector outputs are affected in a similar way. The primary charge collection or the charge multiplication process can be influenced for example by the stability of the power supply (a change of $1 \mathrm{~V}$ on the voltage across both GEMs results in a $1 \%$ variation in the detector response), and small changes in the purity of the gas mixture, in the gas mixture ratio or in the flow rate.

We have observed that the detector output reaches the equilibrium after about three beam shots (of $20 \mathrm{~s}$ and $1 \mathrm{~Gy}$ ). When during measurements the power supply 'trips' (because of an over current) and the GEMs voltages drop to zero and must be raised again, a difference of a few percent in the detector output was found for the first couple of measurements after this operation, with respect to the measurements performed under equilibrium conditions, due to the fact that the detector had to be 'recharged' (Bouclier et al 1997). This contributed to the shown response reproducibility as well.

In the following, the short-term response reproducibility is used as uncertainty of $q_{\text {out }}$ and $L$ for measurements performed in different moments, e.g. days apart.

\subsection{Light signal brightness}

In figure 4, the intensity of the scintillating GEM light signal as a function of the voltage across the GEMs is graphed together with the measured scintillating Lanex screen signal intensity. 


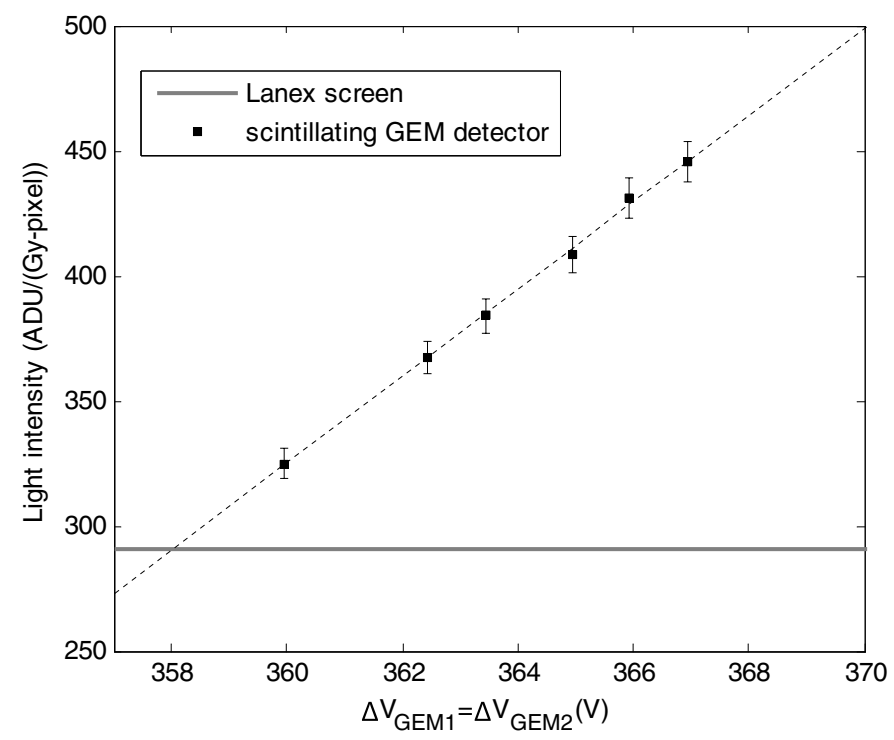

Figure 4. Scintillating GEM light signal intensity as a function of the voltage across the GEMs with $\Delta V_{\mathrm{GEM} 1}=\Delta V_{\mathrm{GEM} 2}$ compared to the scintillating Lanex screen response intensity measured for the same conditions. Small hole GEMs in $\mathrm{Ar}+8 \% \mathrm{CF}_{4}$ were used. Error bars represent $3 \sigma$.

Both experiments were performed delivering $10 \mathrm{~Gy}$, at $30 \mathrm{~Gy} \mathrm{~min}^{-1}$, for a circular $20 \mathrm{~mm}^{2}$ field size at 0 -wd. Small hole GEMs in $\mathrm{Ar}+8 \% \mathrm{CF}_{4}$ were used.

The GEM light signal is brighter than the Lanex screen signal when $\Delta V_{\mathrm{GEM} 1}=\Delta V_{\mathrm{GEM} 2}>$ $358 \mathrm{~V}$. Most of the measurements reported in the following were performed at $\Delta V_{\mathrm{GEM} 1}=$ $\Delta V_{\mathrm{GEM} 2}=370 \mathrm{~V}$. At this tension, the GEM light signal is about 1.4 times higher than that of the Lanex screen.

\subsection{Proton dose-response characterization}

In the upper graph of figure $5, L$ is shown as a function of dose. The latter was varied by changing the amount of delivered dose (MU) for a fixed dose rate of $17.5 \mathrm{~Gy} \mathrm{~min}^{-1}$ and a field size of $11 \times 12 \mathrm{~mm}^{2}$. The measurements were performed with big hole GEMs in $\mathrm{Ar}+$ $6 \% \mathrm{CF}_{4}$.

$L$ is linear with the dose in the investigated range $1-18 \mathrm{~Gy}$, since the relative residuals of the least-squares linear fit are very small. The same good dose linearity was observed for $q_{\text {out }}$.

The $L$ and $q_{\text {out }}$ dose linearity for doses smaller than 1.5 Gy was measured for small-hole GEM in $\mathrm{Ar}+8 \% \mathrm{CF}_{4}$, for $20 \mathrm{~mm}^{2}$ circular field size and $2 \mathrm{~Gy} \mathrm{~min}^{-1}$. We have found that according to the relative residuals of a least-squares linear fit, the $L$ and $q_{\text {out }}$ dose linearity between 0.05 and 2 Gy is within $\sim 1 \%$.

So, within the previously given experimental errors (section 2.4 ), the detector response is linear with the dose in the range $0.05-18 \mathrm{~Gy}$.

\subsection{Proton dose rate response characterization}

In figure $6, L$ and $q_{\text {out }}$ are shown as a function of the dose rate together with the signal of the ionization chamber IC used as a reference. The data were measured for $11 \times 12 \mathrm{~mm}^{2}$ field 

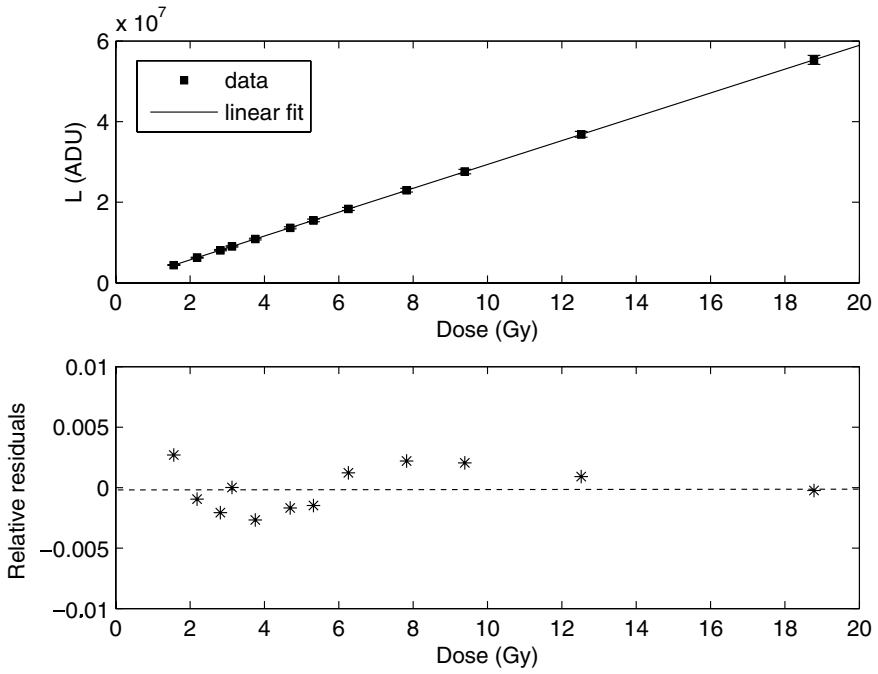

Figure 5. Top graph: $L$ as a function of dose in the range $1.5-19$ Gy measured with big hole GEMs, in $\mathrm{Ar}+6 \% \mathrm{CF}_{4}$. Bottom graph: relative residuals of the least-squares linear fit.

size, a fixed delivered dose of $6 \mathrm{~Gy}$ and big hole GEMs in $\mathrm{Ar}+6 \% \mathrm{CF}_{4}$. For clarity, error bars $(\sim 2 \%, 3 \sigma)$ in the figure are neglected.

The signal $q_{\text {IC }}$ is dose rate independent at both water depths, as expected. At 0 -wd, $L$ and $q_{\text {out }}$ are constant within the experimental errors in the range $1-16 \mathrm{~Gy} \mathrm{~min}^{-1}$. The same is observed for the detector output measured at the Bp-wd in the range $2-38 \mathrm{~Gy} \mathrm{~min}^{-1}$. Although the random $q_{\text {out }}$ and $L$ fluctuations are slightly bigger than those measured at 0 -wd, the values are still consistent within the experimental uncertainties.

So, the scintillating GEM detector response is independent of the dose rate in the range 1-38 Gy $\mathrm{min}^{-1}$ within the experimental error $(3 \sigma)$ irrespective of the factor 3.5 ionization density difference along the depth-dose curve.

\subsection{Spatial response}

3.5.1. Spatial resolution. The spatial response of the scintillating GEM detector was estimated by evaluating the line spread function (LSF). The picture on the inset of figure 7 was taken with a $1 \times 10 \mathrm{~mm}^{2}$ field-shaping collimator with small hole GEMs in $\mathrm{Ar}+8 \% \mathrm{CF}_{4}$. In the graph of figure 7, the single-pixel-wide light intensity profile taken along the white line, represented on the inset, is shown together with the Lanex screen intensity profile along the same line measured under the same conditions.

According to the literature (van Luijk et al 2001), the Lanex screen has a spatial resolution $(\sigma)$ of about $0.2 \mathrm{~mm}$. Consequently, the light intensity profile measured with the screen is used as the beam profile for calculating the spatial response of the scintillating GEM detector.

We assume that the LSF of the scintillating GEM detector system is described by a Gaussian distribution. Then, we make the convolution of the beam profile (Lanex screen profile) with the assumed LSF. In an iterative process, the parameter $\sigma$ of the GEM detector LSF is varied until the mean absolute difference of the convolved reference profile and the scintillating GEM detector profile is minimized. In this way, we found that the scintillating GEM detector LSF has a $\sigma$ of $0.8 \mathrm{~mm}$. 

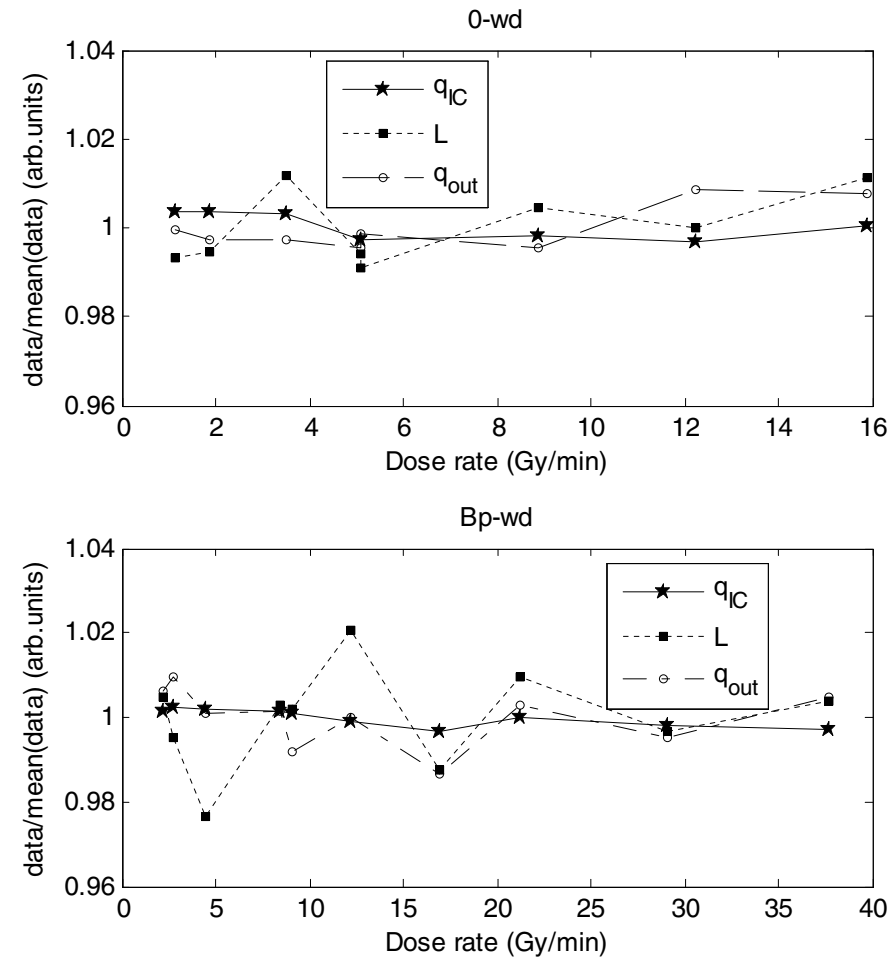

Figure 6. $q_{\mathrm{IC}}, L$ and $q_{\mathrm{out}}$ as a function of the dose rate for 0 -wd (top graph) and Bp-wd (bottom graph). On the $y$-axis, the ratio of the single data values and the average of all data values are shown. For visualization purposes, the experimental error bars $(\sim 2 \%, 3 \sigma)$ are neglected.

3.5.2. Response uniformity. The GEM detector response uniformity was verified studying the light intensity profiles of pictures measured in a homogeneous irradiation field of an $80 \mathrm{~mm}$ diameter circular collimator. An example of a picture taken under such conditions with the scintillating GEM detector, equipped with small hole GEMs in $\mathrm{Ar}+8 \% \mathrm{CF}_{4}$, is shown in figure 8. Some non-uniformities in the intensity of the emitted light can be seen, below the beam spot center and near its edges.

A Lanex signal is taken in this case as a reference since in Boon (1998a) it is shown that its response uniformity is comparable to that of a radiographic film. By normalizing a GEM detector picture to that of the Lanex screen, measured for identical beam conditions and properly rescaled in terms of $L$ to compensate for the signal brightness difference (figure 4), variations in the light magnitude due to common causes like a non-uniform beam and optical distortions can be eliminated. The remaining deviation of the detector response from the Lanex screen response, represented in the bottom graph of figure 9 along a one-pixelwide horizontal line passing by the center of the beam sport, is then the non-uniform response of the scintillating GEM detector itself. Similar variations as those shown in the top graph of figure 9 were found in light-intensity profiles on pictures taken for $70 \mathrm{~mm}, 60 \mathrm{~mm}$ and $50 \mathrm{~mm}$ diameter circular collimators for the same measurement conditions.

The inhomogeneities in the GEM detector emitted light can be due to non-uniform gain over the GEM surface (Bouclier et al 1997), (Yu et al 2003) caused by, for example, hole-shape variations during the manufacturing process (Fraga et al 2000) or a non-uniformly stretched 


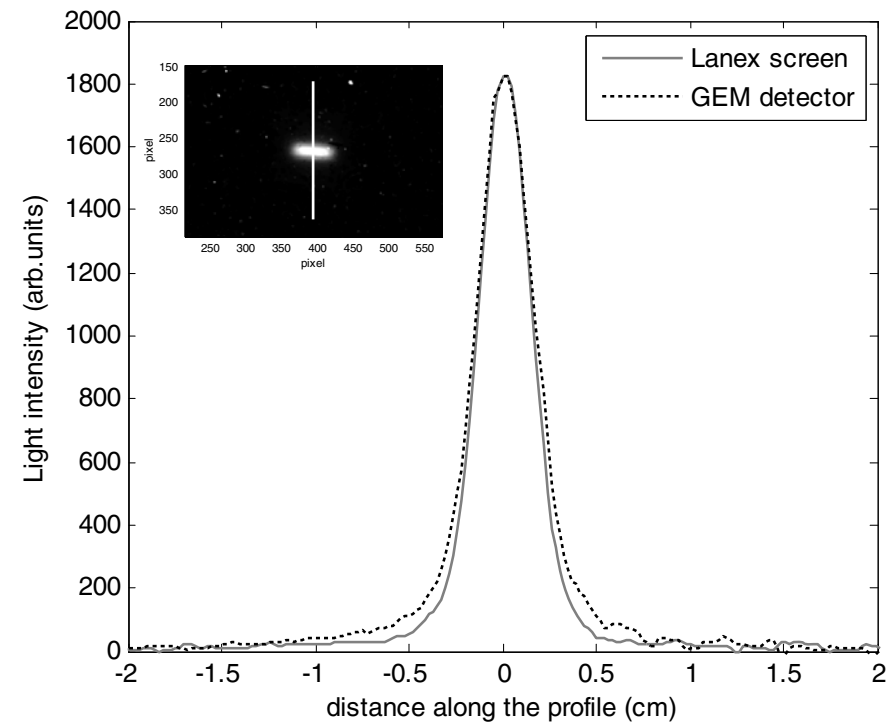

Figure 7. Scintillating GEM detector light intensity profile along the one-pixel-wide white line represented on the inset together with a normalized Lanex light intensity profile measured under the same conditions. Inset: an example of scintillating GEM detector picture taken with a $1 \times$ $10 \mathrm{~mm}^{2}$ field-shaping collimator $(1 \mathrm{~cm}=46$ pixels $)$. GEMs with small holes in $\mathrm{Ar}+8 \% \mathrm{CF}_{4}$ were used for this measurement.

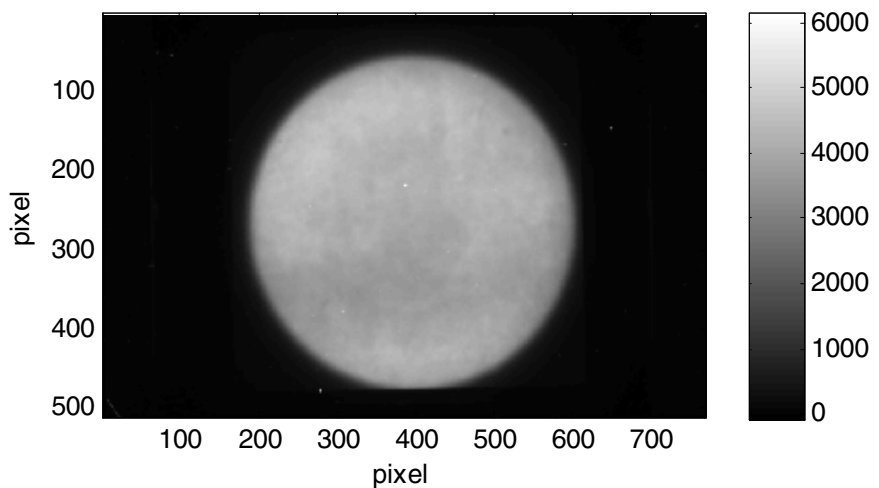

Figure 8. Example of picture taken with the scintillating GEM detector for an $80 \mathrm{~mm}$ diameter circular field-shaping brass collimator $(1 \mathrm{~cm}=46$ pixels).

GEM when it is glued onto the frame. Then, the non-uniformity of the response is stable, but different, for each particular GEM mounted inside the detector.

In that case, the non-uniformity of the response can be corrected for in the following ways. First, a picture with the voltage difference across the GEMs equal to the one used during the measurements is taken for a large field-shaping collimator $(80 \mathrm{~mm} \varnothing)$ together with a reference detector picture (i.e. Lanex screen or film) for identical beam conditions. Then, a matrix of pixel-wide correction factors is obtained by normalizing the scintillating GEM picture to the properly rescaled reference detector picture, in order to have the same 

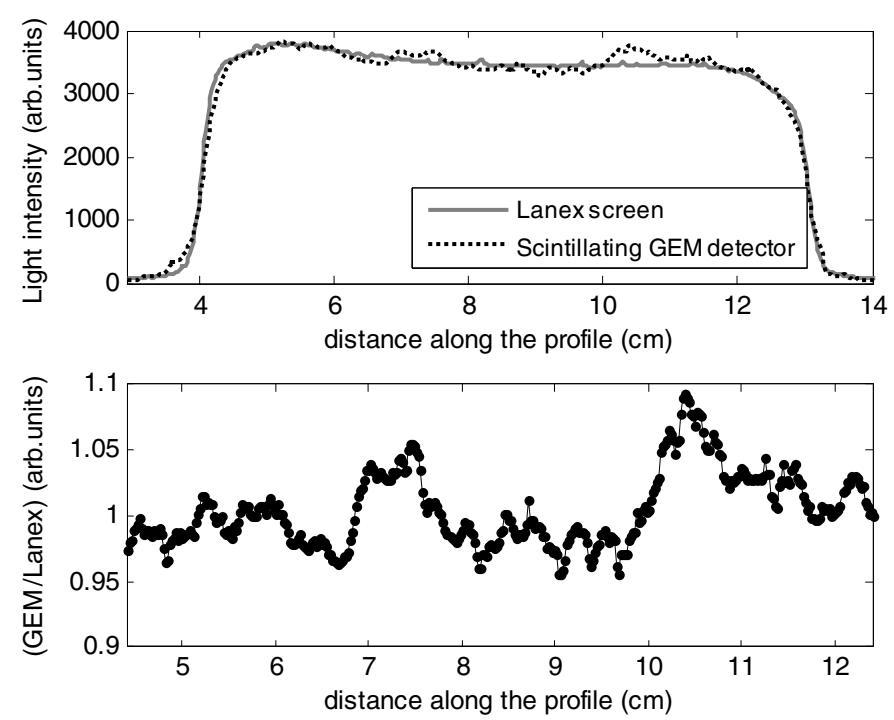

Figure 9. Top graph: scintillating GEM detector and Lanex screen light intensity profiles along the same single-pixel-wide horizontal line, passing by the beam spot center, of the picture taken in the homogeneous irradiation field of an $80 \mathrm{~mm}$ diameter circular collimator. The Lanex light intensity profile was rescaled in order to have the same integrated light yield as the scintillating GEM detector picture. The Lanex profile represents the beam profile, which, however, has not been optimized to make it extremely flat. Bottom graph: ratio of scintillating GEM detector and Lanex light intensity along the profile. The horizontal axis is in this case restricted to the collimator size.

$L$ as the GEM detector picture. Finally, in order to get pictures compensated for the nonuniformity response variations, the scintillating GEM detector pictures are divided by the resulting correction matrix.

3.5.3. Proton field size response characterization. The scintillating GEM detector response as a function of the field size has been investigated by measuring the detector response for several field-shaping brass collimators of different aperture size $\left(11 \times 12 \mathrm{~mm}^{2}, 20 \times 20 \mathrm{~mm}^{2}\right.$ and circular ones with diameters of 30, 50, 60 and $70 \mathrm{~mm}$ ). The experiment was performed for $5 \mathrm{~Gy}$ delivered dose, $6 \mathrm{~Gy} \mathrm{~min}^{-1}$ and at 0 -wd. GEMs with small holes were mounted in the detector chamber that was flushed with $\mathrm{Ar}+8 \% \mathrm{CF}_{4}$.

No evidence of field size dependence of the scintillating GEM detector response was found within the $\sim 2 \%(3 \sigma)$ experimental error in the range $120-3850 \mathrm{~mm}^{2}$.

\subsection{Time response}

In figure 10, the rise ${ }^{5}$ (left graph) and fall time (right graph) of the scintillating GEM detector light signal are shown, together with the IC current signal.

In this case, the GEM light signal was recorded by means of a Hamamatsu R943-02 photomultiplier tube. The output current of the photomultiplier tube and the IC signal were

5 The rise time is defined as the time required for the signal to increase from $10 \%$ up to $90 \%$ of the signal height. The fall time is, respectively, the time needed for a signal decrease from $90 \%$ down to $10 \%$ of the height. 

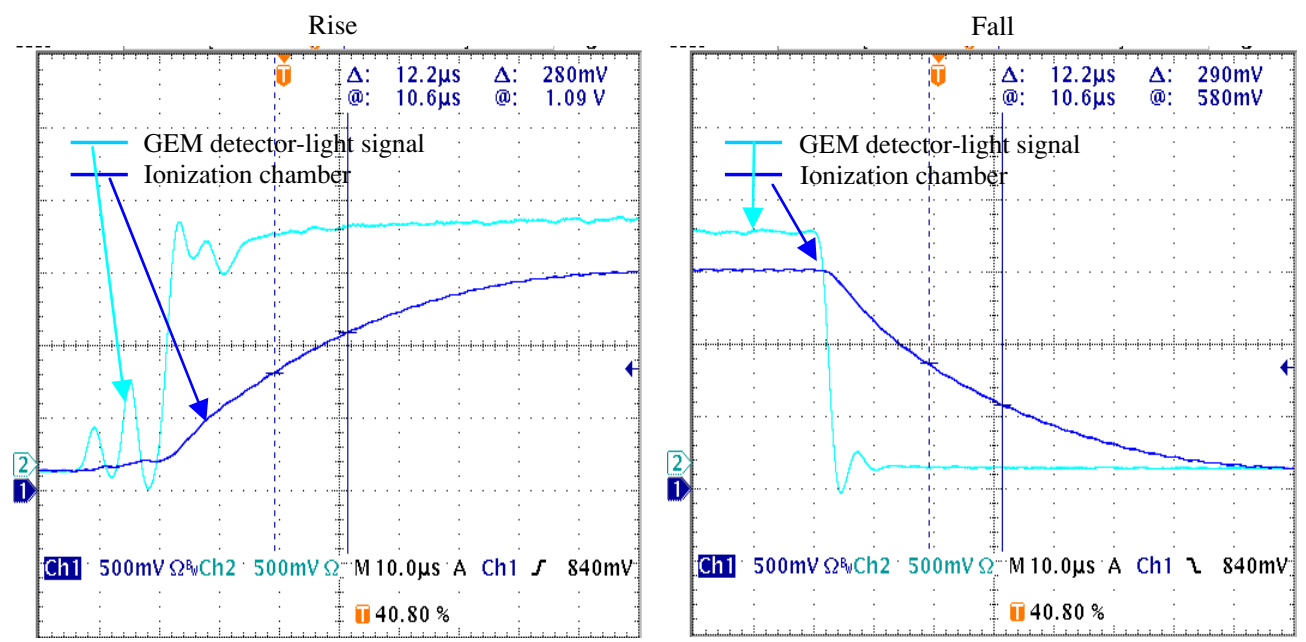

Figure 10. Rise time (left graph) and fall time (right graph) of the scintillating GEM detector light signal (light continuous line) detected by means of a photomultiplier tube fed into an oscilloscope. The IC signal (dark continuous line) is graphed for comparison.

(This figure is in colour only in the electronic version)

observed by means of an oscilloscope. GEMs with big holes were mounted inside the detector chamber that was flushed with $\mathrm{Ar}+6 \% \mathrm{CF}_{4}$.

The signal of the IC is characterized by the large RC time of the electronics. The rise and fall time of the GEM detector light signal is of the order of $2 \mu \mathrm{s}$. This is probably dominated by the rise time of the beam (Brandenburg 2008). In fact, the scintillating GEM detector light response rise time is expected to be faster since the electron drift time in $3.2 \mathrm{~mm}$ drift gap and in $\mathrm{Ar}+10 \% \mathrm{CF}_{4}$ is about $0.05 \mu \mathrm{s}$ (Peisert and Sauli 1984). Similar experiments were performed with the scintillating Lanex screen but then a rise and fall time of about $1 \mathrm{~ms}$ were measured.

The oscillations measured in the GEM detector light signal before the beam start (left graph of figure 10) are likely due to the beam structure because they are also visible in the ionization chamber signal with a lower magnitude. The oscillations just after the beam started (left graph of figure 10) and stopped (right graph of figure 10) are attributed to the electronics used to record the GEM detector light signal.

\subsection{Pulsed beam feasibility study}

In order to check if the GEM detector could work in a pulsed beam or in a scanning beam, a feasibility study was made recording the detector outputs for a different number of beam pulses.

The duration and the number of the beam pulses were varied from measurement to measurement in such a way to have the same delivered total dose, of about $10 \mathrm{~Gy}$, per measurement and the same dose rate, of about $15 \mathrm{~Gy} \mathrm{~min}^{-1}$. The CCD camera exposure time was also the same for all the pictures. Small hole GEMs were used and the detector was flushed with $\mathrm{Ar}+8 \% \mathrm{CF}_{4}$. The field size was a circle of $20 \mathrm{~mm}^{2}$.

In table 2 , an overview of the results is given. $L$ is normalized to $q_{\mathrm{IC}}$ in order to be sure that the comparison is made between values obtained for the same delivered dose. No difference 
Table 2. Study of pulsed beam response for a dose rate of about $15 \mathrm{~Gy} \mathrm{~min}^{-1}$ and a total delivered dose of about $10 \mathrm{~Gy}$. The first column from the left indicates the number of beam pulses or shots delivered per measurements, the second the pulse duration and the third the time between pulses. The uncertainty associated with $L / q_{\mathrm{IC}}$ is the experimental error found to be $2 \%(3 \sigma)$ in this series of measurements.

\begin{tabular}{lllll}
\hline Number of pulses & Pulse duration (s) & Time between pulses $(\mathrm{s})$ & Dose per pulse $(\mathrm{Gy})$ & $L / q_{\text {IC }}($ arb. units $)$ \\
\hline 1 & 41 & - & 10.25 & $(1.14 \pm 0.02) \times 10^{5}$ \\
41 & 1 & 0.1 & 0.25 & $(1.14 \pm 0.02) \times 10^{5}$ \\
400 & 0.1 & 0.1 & 0.025 & $(1.14 \pm 0.02) \times 10^{5}$ \\
4000 & 0.01 & 0.01 & 0.0025 & $(1.17 \pm 0.02) \times 10^{5}$ \\
40000 & 0.001 & 0.005 & 0.00025 & $(1.17 \pm 0.02) \times 10^{5}$ \\
\hline
\end{tabular}

in the detector output is observed, within the experimental errors $(3 \sigma)$, if the 10 Gy dose is delivered in a single pulse or in a number of pulses of shorter duration and smaller Gy content. Similar behavior of $q_{\text {out }}$ as a function of pulse number and dose per pulse was found.

The same study of table 2 was performed for a dose rate, of about $240 \mathrm{~Gy} \mathrm{~min}^{-1}$, close to the one used in proton scanning beams for clinical purposes (Lomax 2007). The total delivered dose per measurement was in this case about $20 \mathrm{~Gy}$. Also in this case, all the $L$ values normalized to the $q_{\mathrm{IC}}$ are comparable within the experimental uncertainty.

\subsection{Tissue equivalence}

When the dose is reported as 'dose to water' (or 'dose to air'), the ratio of the stopping power of the detector material relative to the stopping power in water (or air) has to be taken into account.

For a detector that is not tissue-equivalent, the detector material-to-water stopping power ratio is not one in the particle energy range of interest for radiation therapy. Moreover, the stopping power ratio is not constant as, for example, the air-to-water stopping power ratio.

Although it is still not tissue equivalent, the $\mathrm{Ar} / \mathrm{CF}_{4}$-to-water stopping power ratio varies less than the Lanex screen-to-water ratio in the energy range 1-300 MeV (NIST 2005). This may cause a signal reduction of about $2 \%$ in the Bragg peak, where the proton energy is lower than $10 \mathrm{MeV}$.

\section{Conclusions}

We have developed a dosimetry system for 2D dose measurements in charged particle beams used for cancer treatment. The system consists of a position-sensitive scintillating gas detector, equipped with two cascaded GEMs in an $\mathrm{Ar} / \mathrm{CF}_{4}$ mixture. The photons emitted by the $\mathrm{Ar} / \mathrm{CF}_{4}$ electron-excited molecules, during the gas multiplication process, are detected by a CCD camera coupled to a mirror lens.

In this paper, we described the experiments performed in a $150 \mathrm{MeV}$ proton beam to study those properties that are of particular importance in relative dose measurements.

We found that the detector response $(\sigma=0.6 \%)$ is very stable on measurements performed in succession.

The short-term reproducibility of the detector outputs is about $5 \%$ over 2 days. Causes of this poor reproducibility are not yet understood. They are probably related to the signal formation process since they affect both light and charge response in a similar way. Dynamic detector gain shifts due to charge up effects could also be responsible for response variations 
with time. The short-term reproducibility should be further characterized, especially in anticipation of a clinical usage of the detector.

We found that for small hole GEMs in $\mathrm{Ar}+8 \% \mathrm{CF}_{4}$, and $\Delta V_{\mathrm{GEM} 1}=\Delta V_{\mathrm{GEM} 2}>358 \mathrm{~V}$ the scintillating GEM detector light signal is 1.4 times brighter than the Lanex screen scintillating signal.

A dose linearity of the detector response within $1 \%$ uncertainty was measured for doses between 0.05 and $19 \mathrm{~Gy}$. No dose rate effects were observed at the shallow depth of the water bellows phantom in the range $1-16 \mathrm{~Gy} \mathrm{~min}^{-1}$, and at the Bragg peak depth in the range 2-38 Gy $\min ^{-1}$ within the $\sim 2 \%(3 \sigma)$ experimental uncertainties.

It was found that the scintillating GEM detector line spread function has a $\sigma$ of $0.8 \mathrm{~mm}$. The spatial resolution of the detector was expected to be at the sub-millimeter level because of the low $\mathrm{Ar} / \mathrm{CF}_{4}$ diffusion coefficient and of the high degree of granularity of the GEM holes and of the CCD camera.

The uniformity of the scintillating GEM detector light response was found to be not as good as the Lanex screen. However, the non-uniformity can be compensated for by normalizing the light intensity values of each picture against the picture that results from the ratio of a picture taken for a large field-shaping collimator for the same measurement conditions (type of GEM hole, voltage across the GEMs, gas mixture) and one with the Lanex screen.

No field size effects were observed in the range $120-3850 \mathrm{~mm}^{2}$ within the $\sim 2 \%(3 \sigma)$ experimental uncertainties.

The GEM detector light signal has a rise and fall time of about $2 \mu \mathrm{s}$, which is much faster than the scintillating screen signal $(1 \mathrm{~ms})$. This $2 \mu$ s rise time is probably determined by the beam rise time since from the literature it is known that the GEM detector signals are much faster.

A feasibility study was performed to check if the detector could work in pulsed beam, in particular at dose rates typical for proton scanning beams. No major differences were observed in the detector outputs if $10 \mathrm{~Gy}$ at $15 \mathrm{~Gy} \mathrm{~min}^{-1}$ were delivered in one single pulse or different shorter pulses for the same CCD camera exposure time. The same is concluded for a total dose of $20 \mathrm{~Gy}$ at $240 \mathrm{~Gy} \mathrm{~min}^{-1}$ (typical clinical scanning beam dose rate).

In conclusion, the scintillating GEM detector looks promising as a dosimeter for relative 2D dose measurements in charged particle beams for radiotherapy. The detector response is linear with the dose and it does not present dose rate effects. The detector is able to deal with high intensities, $\sim 10^{8}$ particles $\left(\mathrm{cm}^{2} \mathrm{~s}\right)^{-1}$. Given the sub-millimeter resolution, the scintillating GEM detector allows for 2D verification of complex dose distributions in charged particle treatments with their possible steep dose gradients. The fast detector response opens the possibility for online monitoring of a $2 \mathrm{D}$ scanning beam as well with a CCD readout adapted for fast data acquisition.

\section{Acknowledgments}

This work was performed in the framework of the European Integrated Project MAESTRO (Methods and Advanced Equipment for Simulation and Treatment in Radio-Oncology) which is granted by the European Commission under contract number LSHC-CT-2004-503564.

\section{References}

Boon S N 1998a Dosimetry and quality control of scanning proton beams PhD Thesis University of Groningen, The Netherlands 
Boon S N, van Luijk P, Böhringer T, Coray A, Lomax A, Pedroni E, Schaffner B and Schippers J M 2000 Performance of a fluorescent screen and CCD camera as a two-dimensional dosimetry system for dynamic treatment techniques Med. Phys. 27 2198-208

Boon S N, van Luijk P, Schippers J M, Meertens H, Denis J M, Vynckier S, Medin J and Grusell E 1998b Fast 2D phantom dosimetry for scanning proton beams Med. Phys. 25 464-75

Bouclier R, Dominik W, Hoch M, Labbé J C, Million G, Ropelewski L, Sauli F, Sharma A and Manzin G 1997 New observations with the gas electron multiplier (GEM) Nucl. Instrum. Methods A 396 50-66

Brandenburg S 2008 Private communication

Butson M J, Yu P K N, Cheung T and Metcalfe P 2003 Radiochromic film for medical radiation dosimetry Mater. Sci. Eng. R 41 61-120

Fetal S, van Eijk C W E, Fraga F, de Haas J, Kreuger R, van Vuure T L and Schippers J M 2003 Dose imaging in radiotherapy with an $\mathrm{Ar}-\mathrm{CF}_{4}$ filled scintillating GEM Nucl. Instr. Methods A 513 42-6

Fraga F A F, Fetal S T G, Ferreira Marques R and Policarpo A J P L 2000 Quality control of GEM detectors using scintillation techniques Nucl. Instrum. Methods A 442 417-22

Hamamatsu 2008 Application note http://learn.hamamatsu.com/articles/ccdsnr.html

Holmes-Siedle A and Adams L 1993 Handbook of Radiation Effects (Oxford: Oxford University Press) p 242

KVI website 2008 http://www.kvi.nl/ agorcalc/agorhome.htm

Lomax A J 2007 Private communication

MathWorks 2007 Matlab medfilt2 routine, MATLAB ${ }^{\mathbb{E}}$ - The Language of Technical Computing, version 7.0.0.19920 (R14)

Murray R B and Meyer A 1961 Scintillating response of activated inorganic crystals to various charged particles Phys. Rev. 122 815-26

NIST 2005 http://physics.nist.gov/PhysRefData/Star/Text/contents.html

Peisert A and Sauli F 1984 Drift and diffusion of electrons in gases a compilation CERN Report 84-08

Safai S, Lin S and Pedroni E 2004 Development of an inorganic scintillating mixture for proton beam verification dosimetry Phys. Med. Biol 49 4637-55

Sauli F 1997 GEM: a new concept for electron amplification in gas detectors Nucl. Instrum. Methods A 386 531-4

Seravalli E 2008a A scintillating GEM detector for 2D dose imaging in hadron beams PhD Thesis IOS Press, Amsterdam

Seravalli E, de Boer M, Geurink F, Huizenga J, Kreuger R, Schippers J M and van Eijk C W E 2008b A scintillating gas detector for 2D dose measurements in clinical carbon beams Med. Phys. Biol 53 4651-65

Seravalli E, de Boer M, Geurink F, Huizenga J, Kreuger R, Schippers J M and van Eijk C W E 2008c Characterization of a scintillating gas detector in an x-ray beam Med. Phys. Biol 53 6195-209

Seravalli E, Hendrikse J, Huizenga J, Kreuger R, Schippers J M, Simon A and van Eijk C W E 2007 First results of a scintillating GEM detector for 2D dosimetry in an alpha beam IEEE Trans. Nucl. Sci. 541271

Spielberger B, Kramer M and Kraft G 2003 Three-dimensional dose verification with x-ray films in conformal carbon ion therapy Phys. Med. Biol. 48 497-505

Spielberger B, Scholz M, Kramer M and Kraft G 2001 Experimental investigations of the response of films to heavy-ion irradiation Phys. Med. Biol. 46 2889-97

Timmer J H, van Vuure T L, Bom V, van Eijk C W E, de Haas J and Schippers J M 2002 A scintillating GEM for 2D-dosimetry in radiation therapy Nucl. Instrum. Methods A 478 98-103

van Luijk P et al 2001 Collimator scatter and 2D dosimetry in small proton beams Phys. Med. Biol. 46653

Vukolov K Y and Levin B A 2003 Results of irradiation tests of KU-1 and KS-4V silica glasses as ITER candidate window materials Fusion Eng. Des. 66-68 861-4

Yu B et al 2003 Study of GEM characteristic for application in Micro TPC IEEE Trans. Nucl. Sci. 50836 